\title{
Régénération en altitude de l'épicéa (Picea abies (L) Karst) sur les souches dans les Alpes françaises
}

\author{
P Gensac \\ Laboratoire de pédologie biologique, université de Savoie, \\ BP 1104, 73011 Chambéry Cedex, France
}

(Reçu le 25 avril 1988 ; accepté le 28 avril 1989)

\begin{abstract}
Résumé - La régénération sur souches et bois pourrissants ayant été constatée depuis longtemps dans les forêts d'épicéa d'altitude où le renouvellement est très difficilement assuré. de nouvelles précisions sont apportées sur l'installation et la croissance des jeunes plants d'épicéa dans ces conditions. Après une analyse bibliographique, la localisation des recherches - basse et moyenne Tarentaise - et les méthodes employées - transects de $10 \times 100 \mathrm{~m}$ - sont précisées. Les résultats des observations sont fournis de façon détaillee pour les souches de coupe suivant 5 classes correspondant à une dégradation progressive. Après une implantation maximale des jeunes semis, une disparition progressive des plants est constatée. L'évolution des souches et leur capacité à héberger de jeunes épicéas sont différentes suivant les conditions écologiques : la capacité est très faible dans la pessière à hautes herbes, faible dans les pessières à myrtille, conséquente dans la pessière montagnarde d'ubac à mélampyre et dans la pessière-sapinière d'adret. Pour parvenir à dégager des techniques sylvicoles appropriées aux forêts de montagne, des expérimentations ont été entreprises.
\end{abstract}

\section{Picea abies / Alpes françaises septentrionales / régénération / forêt d'altitude / souche / bois pourri / condition écologique}

Summary - High-altitude regeneration of spruce on old stumps in the French Alps. Regeneration of spruce forest is very difficult above an elevation of $1,500 \mathrm{~m}$. However, young saplings can often be observed growing on old stumps. The aim of the present study is to provide new information on the decomposition of wood and its colonisation by plants, particularly young trees. A literature review is provided and the area under study is detailed: the middle and high Tarentaise valley and the high Isere valley, in the Northern French Alps. The analysis of the forest was carried out on areas measuring $10 \times 100 \mathrm{~m}$, and each stump is reported on the map. The stumps have been sorted into 5 categories according to their degree of decomposition: I, very recent, intact stumps; II, recent stumps, the bark having peeled away with the establishment of the first plants; III, older stumps with a more extensive plant colonisation and considerable establishment of young trees; $V$, old, highly decayed stumps, almost complete plant colonisation and many conifer saplings; $V$, very old and hardly visible stumps, completely covered by vegetation (mainly Vaccinium myrtillus), the saplings 
being less numerous than in category $N$. The development of these 5 stages depends on the ecological conditions prevalling on the site. In spruce forests where there is a high grass cover, the seedlings do not establish on stumps; and where there is bilberry coverage they seldom do so either. At lower elevation spruce forests, they are very often established on stumps, as is the case in forests exposed to the south. In order to facilitate regeneration it is recommended that the forest floor is raked so as to mix wood debris into the soil.

\section{Picea abies / Northern French Alps / high-altitude forest / regeneration / tree-stump / decayed wood / ecological condition}

\section{INTRODUCTION}

Le renouvellement des forêts d'épicéa au-dessus de $1500 \mathrm{~m}$, dans les Alpes françaises septentrionales, paraît difficile à assurer. En effet, dans ces peuplements pratiquement purs, les jeunes arbres, c'est-à-dire d'un âge inférieur à 80 ans, sont rares et les très jeunes sujets sont absents. Ce défaut de régénération conduit inéluctablement, à l'échelle du siècle, à une désagrégation du manteau forestier. II pose le problème de l'équilibre de ces formations d'altitude, problème qui paraît général pour de nombreuses forêts résineuses du globe, exploitées ou non. Comme ce défaut affecte de vastes surfaces selon les services départementaux de l'ONF, plus de 15000 ha dans les Alpes savoyardes -, ses conséquences risquent d'être catastrophiques pour la stabilité des versants et le maintien des activités économiques dans les régions de montagne. L'importance théorique et pratique que présente cette question justifie le choix de l'équipe de pédologie biologique de l'université de Savoie (équipe intégrée à l'Unité associée CNRS $n^{\circ}$ 242), dont le theme unique de recherche est la definition des causes du blocage au renouvellement pour les premiers stades du jeune plant se déroulant à la surface du sol. Cependant, 2 situations paraissent favorables à l'implantation de nouveaux individus dans la zone altitudinale considérée : sur sol minéral brut, d'une part (publication en cours), et sur bois pourris de souches et troncs au sol, objet de cette présentation, d'autre part. De nombreux jeunes arbrisseaux et plantules pouvant être observés sur les souches, il s'agit de préciser leurs conditions d'installation et de survie afin de déterminer leur possible intervention dans les processus de renouvellement des forêts d'altitude. A partir de là, des enseignements pourront être dégagés et ainsi servir à l'élaboration de méthodes sylvicoles appropriées.

La régénération sur souches et troncs pourris a été décrite par Mathey (1911), ce milieu particulier correspondant à un véritable "germinatoire». Cependant, une disparition rapide des semis au bout de 1 à 3 ans peut y être constatée. Cette observation est reprise et précisée par Lachaussée (1947), la neige jouant un rôle déterminant dans l'absence de régénération ainsi que l'humus par son extrême acidite. Duchaufour (1953) reprend la question des "caprices de régénération de l'épicéa", les souches intervenant également par une acidité moindre que celle de l'humus. Par la suite, l'absence de renouvellement des pessières à hautes herbes fait l'objet d'une étude plus précise de Moreau et Poly (1968), qui soulignent le rôle des vieilles souches et des plages de bois pourrissant. Mais ce sont principalement les travaux de Eichrodt 
(1970), dont les conclusions sont reprises par Mayer (1976), qui fournissent le plus d'explications sur la germination et le développement de l'épicéa sur bois pourris, l'hétérogénéité de ce substrat dans l'espace et dans le temps étant favorable à la régénération. Les propriétés fongicides du cœur vis-à-vis des parasites, le régime hydrique, la brièveté du manteau neigeux. l'absence de concurrence des autres végétaux ligneux et herbacés sont autant de facteurs qui faciliteraient, selon ces auteurs, l'installation des jeunes plants d'épicéa dans ces conditions. Maser et Trappe (1984a et b) soulignent également le rôle joué par la diversité structurale des bois tombés dans la régénération de Pseudotsuga menziesii. Plus récemment, Piussi (1986) indique pour les pessières subalpines des Alpes italiennes que, parallèlement à la localisation préférentielle des semis sur terre minérale, on trouve egalement ceux-ci sur les troncs au sol et les souches, soit sur des supports organiques, des explications semblables étant fournies. Enfin, Imbeck et Ott (1987) soulignent que les vieilles souches en putréfaction constituent des stations favorables à la régénération dans les pessières à hautes herbes du canton des Grisons (Suisse)

L'intervention de la mycorhization sur bois pourri présente un intérêt particulier, les travaux en faisant état se trouvant très nombreux. A la suite de l'occupation de souches par les champignons lignivores (Jacquiot, 1978) qui préparent le terrain, les racines des jeunes épicéas pénètrent le substrat, étant alors associées à des champignons mycorhiziens (Goebl, 1968) qui trouvent un excellent refuge dans le bois pourrissant (Boullard, 1969). Ce substrat favorable à l'activité mycorhizienne a été particulièrement étudié par Kropp (1982a et b) qui a suivi l'évolu- tion des jeunes plants de Tsuga heterophylla et y a recherché les types mycorhiziens, ne decouvrant pas de differences significatives dans ce domaine entre bois pourri et sol minéral. Christy et al (1982) ont étudié la survie des jeunes plants de la même espèce et noté le très grand avantage que constitue pour eux la mycorhization. De nombreux travaux ont été effectues par Harvey et al (1976, 1978, 1979, 1980a et b) dans les forêts des montagnes Rocheuses de l'ouest du Montana, où ils ont montré l'étroite dépendance des ectomycorhizes avec la matiere organique du sol, plus spécialement le bois pourrissant, qui offre de meilleures conditions d'humidité en été et permet ainsi le maintien d'une activité mycorhizienne favorable aux plantules de résineux. La grande majorité des semis d'un âge supérieur à 2 ans qui ont été prélevés sur les souches dans les stations d'observation presentaient des mycorhizes.

If apparaît donc nécessaire d'apporter des précisions complémentaires. Les forêts d'épicéa des Alpes du Nord présentant un riche éventail de conditions écologiques, elles ont été choisies pour aborder ce problème.

\section{MATÉRIEL ET MÉTHODES}

\section{Stations}

Les 5 stations d'etude se situent en Tarentaise, haute vallée de l'isère, 4 d'entre elles dans le canton d'Aime, partie moyenne de cette vallée à la limite, pour ce qui concerne la végétation, entre les Alpes intermédiaires et les Alpes internes, la dernière se trouvant en Basse Tarentaise dans les Alpes intermediaires. Le tableau | fournit les caractéristiques de ces stations. Elles se trouvent au-delà de $1500 \mathrm{~m}$ d'altitude et présentent à l'intérieur des pessières differents faciès, 
Tableau I. Caractéristiques des stations étudiées.

\begin{tabular}{|c|c|c|c|c|c|c|}
\hline Station & $\begin{array}{l}\text { Altitude } \\
\text { (m) }\end{array}$ & Exposition & $\begin{array}{c}\text { Type } \\
\text { de pessière }\end{array}$ & $\begin{array}{c}\text { Nombre } \\
\text { d'arbres pour } \\
1000 \mathrm{~m}^{2}\end{array}$ & $\begin{array}{l}\text { Recouvre- } \\
\text { ment } \\
\text { arbres (\%) }\end{array}$ & $\begin{array}{l}\text { Nombre de } \\
\text { souches } \\
\text { inventories }\end{array}$ \\
\hline $\begin{array}{l}\text { MAC B } \\
\text { MAC H } \\
\text { MAC M } \\
\text { COT S } \\
\text { ESS B }\end{array}$ & $\begin{array}{ll}1 & 630 \\
1 & 880 \\
1 & 790 \\
1 & 550 \\
1 & 580\end{array}$ & $\begin{array}{l}\text { N-NO } \\
\text { NE } \\
\text { N-NO } \\
\text { S-SE } \\
\text { SE }\end{array}$ & $\begin{array}{c}\text { à mélampyre } \\
\text { à myrtille I } \\
\text { à hautes herbes } \\
\text { à sapin } \\
\text { à myrtille ॥ }\end{array}$ & $\begin{array}{l}15 \\
36 \\
31 \\
14 \\
19\end{array}$ & $\begin{array}{l}60 \\
75 \\
70 \\
60 \\
70\end{array}$ & \begin{tabular}{r|}
97 \\
73 \\
107 \\
73 \\
65
\end{tabular} \\
\hline
\end{tabular}

dont la typologie a été précisée par Gensac (1967, 1988). On peut les regrouper de la façon suivante :

- étage subalpin inférieur : pessière à myrtille $(\mathrm{MACH})$; pessière à hautes herbes (MAC M) :

- étage montagnard supérieur:

- en exposition nord : pessière à mélampyre (MAC B) ; pessière secondaire à myrtille (ESS B) ;

- en exposition sud : pessière à sapin (COT S)

Pour MAC H, MAC B et ESS B, Vaccinium myrtillus constitue un tapis presque continu, les hautes herbes Adenostyles alliariae et $\mathrm{Ci}$ cerbita alpina dominant très largement dans MAC $M$, alors que le tapis herbacé est très varié dans COT $S$. Ces differentes stations font, par ailleurs, l'objet d'observations et d'expérimentations dans le cadre de recherches plus générales sur le renouvellement des forêts d'altitude.

\section{Méthodes}

Pour atteindre les buts recherchés, il fallait disposer d'un nombre suffisant de souches dans chacune des conditions écologiques précédentes. II a donc été choisi d'effectuer un inventaire précis des stations sur des bandes de $10 \mathrm{~m}$ de large et $100 \mathrm{~m}$ de long suivant les courbes de niveau, opération répétee 3 fois par station. Dans chacune de ces bandes, les souches ont été soigneusement repérées et analysées; diamètre et hauteur, ont été mesurés; les qualités des différentes parties (écorce, aubier, cœur) ont été indiquées, la colonisation par la végéta- tion muscinale, herbacée, ligneuse et l'implantation des jeunes plants et arbrisseaux dont la hauteur a été notée, ont été situees, le tout en établisant un plan horizontal des différentes données recueillies. L'état des souches pour une station correspond bien évidemment à l'âge des travaux de bûcheronnage ou à la chute des troncs, âge qu'il n'a pas été possible de déterminer faute de documents forestiers précis, sauf pour les 2 dernières coupes, 1967 et 1983, de la station MAC B. Cet état dépend également des conditions stationnelles, l'élévation en altitude, à laquelle correspond un ralentissement de l'activité biologique, retardant considérablement la dégradation des bois pourrissants. De même, l'exposition intervient en modifiant les conditions de température et d'humidité. II s'agit donc uniquement d'une étude synchronique des souches sans qu'il ait eté possible de fixer une échelle chronologique exacte des phénomènes, mais il faut souligner que le repérage des surfaces inventoriées autorisera par la suite (dans 20 ou 50 ans...) une recherche diachronique.

\section{RÉSULTATS}

Ils concernent l'évolution des souches, leur colonisation et l'installation corrélative des semis d'épicéa ou autres résineux, suivant les differentes conditions stationnelles.

Dès les premières observations, les souches se sont révélées appartenir a 2 catégories : 
- souches provenant d'un abattage par sciage, présentant une section franche et plate sauf sur la ligne réunissant les 2 attaques où la chute du tronc a provoqué un déchiquetage du bois :

- souches provenant d'un chablis ayant cassé l'arbre à une faible hauteur du sol (de $20 \mathrm{~cm}$ à $1,50 \mathrm{~m}$ ) et n'ayant pas fait l'objet d'un sciage ultérieur, présentant donc une section entièrement déchiquetée. Les régénérations sur souche de chablis étant extrêmement rares, ce cas ne sera pas examiné ici.

\section{La dégradation des souches}

Seuls, jusqu'à présent, les troncs au sol ont fait l'objet de classifications suivant leur degré de dégradation, et c'est donc parallèlement à celles-ci qu'a pu être établie la classification des souches. Les travaux de Mac Cullough (1948) sur les forêts de Picea enge/manii el Abies lasiocarpa aboutissent à une échelle d'évolution de 1 (arbre de chute récente) à 8 (où le pourrissement est complet), la colonisation par les végétaux étant nettement précisée alors que l'établissement des jeunes plants paraît indépendant de la succession. Les différents stades de dégradation des bois tombés au sol ont été plus récemment décrits par Maser et al (1979) qui, reprenant une classification établie par Mac Millan et al (1977), aboutissent en 1984 à 5 classes pour les troncs de Pseudotsuga menziesii. Cette dernière classification a donc été adaptée au cas des souches de coupe d'épicéa et aussi éventuellement de mélèze (tableau II). La distinction des classes I, II et III est basée principalement sur les caractères de l'écorce, alors que les dernières classes sont différentes par leur degré de pourriture, l'épaisseur et l'évolution de la litière, la colonisation qui est complète dans la classe $\mathrm{V}$.

\section{Leur colonisation progressive par la végétation (fig 1c)}

Elle s'effectue progressivement depuis la classe I, où la coupe est trop récente pour présenter le moindre végétal visible, jusqu'à la classe V. La figure 1c fournit les classes de fréquence des différentes espèces.

Tableau II. Différentes classes de décomposition des souches et leurs caractères de reconnaissance.

\begin{tabular}{|c|c|c|c|c|c|}
\hline \multicolumn{6}{|c|}{ Classe } \\
\hline & 1 & 11 & III & IV & $V$ \\
\hline Agé en MAC B & 6 ans & 20 ans & 40 ans & $?$ & $?$ \\
\hline Ecorce & en place & $\begin{array}{l}\text { décollée, per- } \\
\text { sistante autour }\end{array}$ & absente en partie & absente & absente \\
\hline Aubier & dur & $\begin{array}{l}\text { Friable en } \\
\text { periphérie }\end{array}$ & $\begin{array}{l}\text { friable sous } \\
\text { forme de prisme }\end{array}$ & $\begin{array}{c}\text { friable } \\
\text { pulvérulent }\end{array}$ & pourri \\
\hline Cceur & dur & dur & dur & début pourriture & $\begin{array}{l}\text { pourriture brun- } \\
\text { rouge ou grise }\end{array}$ \\
\hline Litiere & absente & peu abondante & $\begin{array}{l}\text { mince et non } \\
\text { fermenté }(\mathrm{OL})\end{array}$ & $\begin{array}{l} \pm \text { épaisse en trans } \\
\text { formation }(\mathrm{OF})\end{array}$ & $\begin{array}{l}\text { abondante } \\
\text { humification }\end{array}$ \\
\hline $\begin{array}{l}\text { Colonisation } \\
\text { par végétation }\end{array}$ & absente & très faible & faible & sur les $3 / 4$ & $\begin{array}{c}(\mathrm{OH}) \\
\text { complète }\end{array}$ \\
\hline
\end{tabular}




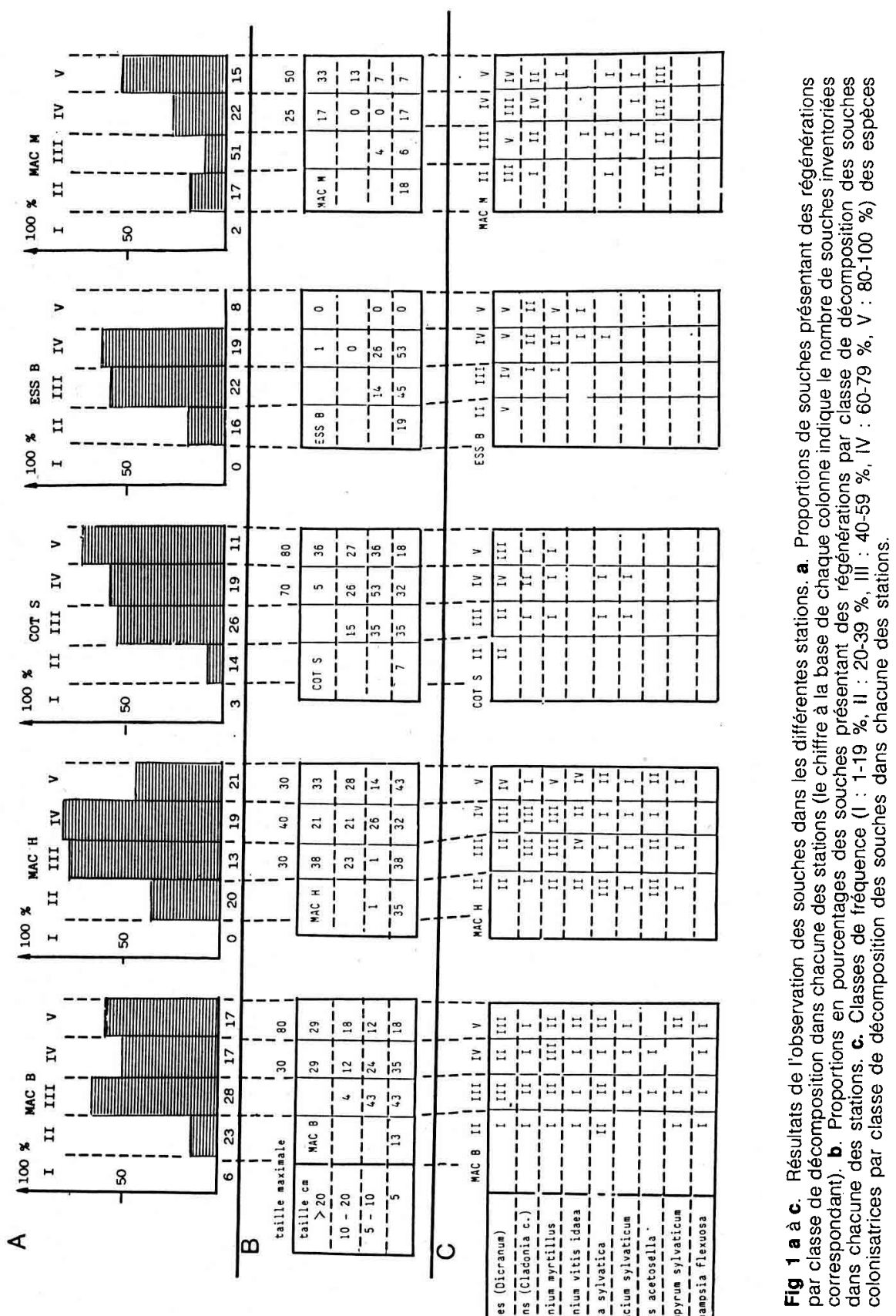


Très généralement, les mousses (dans la grande majorité des cas Dicranum scoparium) forment le premier peuplement, se développant en surface pour parvenir à un recouvrement total dans la classe V. Dans les premiers stades, elles sont fréquemment accompagnées de lichens, Cladonia coniocrea étant l'espèce de loin la plus abondante, cette lignicole régressant par la suite.

Les Vacciniees, surtout Vaccinium myrtillus, présentent des fréquences élevées sur les souches, principalement dans les derniers stades de décomposition de MAC $H$ et ESS B (les 2 pessières à myrtille). L'efficacité de leur reproduction par graines sur les souches est à opposer à leur rapide extension par rhizomes sur le sol forestier. Pour les classes $V$ de décomposition, elles peuvent atteindre leur taille maximale de $40 \mathrm{~cm}$ de hauteur et un très grand recouvrement. Elles sont pratiquement absentes de la pessière d'adret (COT $S$ ) et de la pessière à hautes herbes (MAC M).

Les autres espèces, Luzula sylvatica, Hieracium sylvaticum, Melampyrum sylvaticum et Deschampsia flexuosa, sont surtout présentes dans MAC $H$ et MAC B, pessières internes sans hautes herbes, alors qu'Oxalis acetosella est abondante dès les premiers stades dans MAC M (pessière à hautes herbes).

\section{L'installation des résineux à leur surface}

D'une façon globale, sans distinguer la taille de la régénération, on peut constater que la colonisation des souches par les résineux (dans la grande majorité des cas par l'épicéa) s'effectue de façon très différente suivant les conditions écologi- ques (fig 1a). Sur les 309 souches inventoriées au total, 164 , soit $41 \%$, présentent des régénérations, le taux le plus élevé se situant en MAC $\mathrm{H}$, pessière à myrtille supérieure (56\%), le plus bas en MAC M, pessière à hautes herbes (22\%).

En prenant en compte la taille des régénérations par distinction de 4 catégories (fig 1b), on peut constater que leur implantation, stade plantule ou plant de 2 ans $(<5 \mathrm{~cm})$, s'effectue à partir de la classe II de decomposition et ce de façon très constante tout au long de l'évolution de la souche, sauf pour ESS B, pessière à myrtille secondaire, où aucune des 8 plus vieilles souches ne présente de plantules, tout en signalant la faiblesse du nombre d'observations dans ce cas. Pour les stades ultérieurs, l'absence de régularité dans la croissance des tailles au fur et à mesure du vieillissement de la souche est évidente. Elle peut être due soit à une mortalité affectant les régénérations selon les circonstances climatiques, soit à des arrêts ou ralentissements de la croissance durant plusieurs années. Sauf pour la pessière à hautes herbes (MAC M) où le taux de régénération est le plus faible, on peut constater que le taux des souches de classe $V$ présentant des plants $>20 \mathrm{~cm}$ est inférieur à celui que l'on pourrait attendre de la croissance des plants installés antérieurement sur les souches. Sur l'ensemble des $3000 \mathrm{~m}^{2}$ inventoriés dans chaque station, le nombre de souches de classe $\checkmark$ portant des plants $>20 \mathrm{~cm}$ est compris entre 4 et 7 , soit moins de 24 par ha dans le meilleur des cas.

Une comparaison s'impose dans chaque station, entre la régénération sur souches et celle s'effectuant en dehors d'elles, sur le sol qui a également fait l'objet de comptages. La pessière 
à sapin, COT S, est la seule des 5 stations a présenter une régénération satisfaisante, plus de 2 jeunes plants ou plantules au $\mathrm{m}^{2}$, en épicéa mais surtout en sapin, un changement dans le peuplement est donc prévisible ; c'est également la station où la régénération sur souches est la meilleure, le nombre et la taille des semis les plus élevés. Vient ensuite MAC B, pessière à mélampyre, où la régénération s'effectue par taches isolées de nombreux semis, mais n'occupant qu'une surface maximale de $20 \%$ à l'intérieur du peuplement, la régénération sur souches s'y trouve encore assez bonne. Pour les 3 autres stations, pessières à myrtille supérieure (MAC $\mathrm{H}$ ) et secondaire (ESS $B)$, pessiere à hautes herbes (MAC M), en dehors des souches, il n'y a pas de jeunes semis.

\section{DISCUSSION}

Dans 4 stations sur 5 , les souches représentent donc bien, comme Mathey (1911) l'indiquait, un lieu privilégié pour l'installation des jeunes plants de résineux. Dès le stade de décomposition correspondant à la classe II, soit 10 à 15 ans après l'abattage pour la station MAC B (pessière a mélampyre), la germination des graines et la croissance de la plantule peuvent s'effectuer. Pour les stades III et IV, où les souches présentent une hétérogénéité maximale, plus de la moitié d'entre elles présente des régénérations plus ou moins développées (sauf pour la station MAC M, pessière à hautes herbes, où ce degré de colonisation n'est atteint qu'en classe V). Ceci est en accord avec les travaux de Maser et Trappe (1984a et b) et ceux de Moreau et Poly (1968) constatant la rareté des régénérations sur souche dans la pessière à hautes herbes. Cependant, il faut bien souligner que la densité de plants dépassant $20 \mathrm{~cm}$ de hauteur, donc susceptibles de persister et de participer au renouvellement de la forêt, est extrêmement faible. Seule la surface de la souche ayant été prise ici en considération, il faut signaler la présence sporadique de jeunes résineux, à proximité immédiate de la base et en relation avec la présence de grosses racines en voie de décomposition. Le matériel ligneux en putréfaction pourrait donc bien être favorable à la régénération, et son apport à un sol au cours d'un crochetage pour accélérer son évolution et activer l'humus brut serait alors un procédé pour traiter par petites surfaces $\left(100 \mathrm{~m}^{2}\right)$ les sites les plus favorables dans les peuplernents affectés par le manque de régénération. Ces matérieux ligneux sont actuellement des sous-produits abondants dont l'élimination pose problème dans nos vallées alpines. Sciures, copeaux, bois rémanants déchiquetés aideraient à moindre prix à résoudre en partie les défauts de renouvellement par création de petits collectifs servant à la restructuration des peuplements en voie de vieillissement. Des expériences sur placettes sont actuellement poursuivies en ce sens par les services de l'ONF, leur mise en place en 1988 ne permet évidemment pas d'évaluer leurs résultats.

\section{REMERCIEMENTS}

Ces travaux se situent dans le prolongement de ceux effectués dans le cadre du programme Piren, "L'aménagement de la haute montagne et ses consequences sur l'environnement, le canton d'Aime (Savoie)". Je remercie J. André d'avoir bien voulu me communiquer l'importante bibliographie qu'il avait rassemblée sur cette question. Mes remerciements vont également aux différents 
agents de l'Office national des forêts, service départemental de la Savoie, cellule technique d'Albertville, pour l'aide effective qu'ils nous apportent.

\section{RÉFÉRENCES}

Boullard B (1969) Importance des mycorhizes pour la régénération de l'épicéa (Picea excelsa Link). Soc For FrancheComté Prov Est mars, 3-11

Brown JL (1977) Étude de la perturbation des horizons du sol par un arbre qui se renverse et de son impact sur la pédogenèse. Can J Soil Sci 57, 173-186

Christy EJ, Sollins P, Trappe JM (1982) Firstyear of Tsuga heterophylla without mycorrhizae and subsequent ectomycorrhizal development on decaying logs and mineral soils. Can $J$ Bot 60 , $1601-1605$

Duchaufour P (1953) Régénération de l'épicéa et pédologie. Rev For Fr (Nancy) 4, 257-268

Eichrodt $R$ (1970) Ueber die Bedeutung von Moderholz fur die natürliche Verjüngung im subalpinen Fichtenwald. Beih $Z$ Schweiz For 45, 122

Froidevaux L. (1975) Dans la réserve de Derborence, un rescapé de l'exploitation des forêts : Poria terrestris (DC exfries Sacc), mycorhizique sur Abies alba, Larix decidua et Picea abies. Schweiz $Z$ Fortswes 126, 65-66

Froidevaux L (1978) Les Hyménomycètes résupinés mycorhiziques dans le bois pourri. Schweiz Z Forstwes 106, 9-14

Gensac P (1967) Les forêts d'épicéa de Moyenne Tarentaise. Recherche des différents types de pessières. Rev Gen Bot $74,425-528$

Gensac P (1988) Types de pessière et régénération en Moyenne Tarentaise (Savoie). Rev For Fr (proposé au Comité de rédaction)

Goebl F (1968) Mycorhiza-Untersuchungen an Jungfichten im Urwald von Brigels. Schweiz Z Forstwes 2, 148-150

Harvey AE (1976) Distribution of Ectomycorrhizae in a mature Douglas fir Larch Forest Soil in Western Montana. For Sci 22, 393-398
Harvey AE (1978) Seasonal Distribution of Ectomycorrhizae in a mature Douglas fir/Larch Forest Soil in Western Montana. For Sci 24, 203-208

Harvey AE (1979) Comparative distribution of Ectomycorrhizae in soils of three Western Montana Forest Habitat types. For Sci $25,350-358$

Harvey AE (1980a) Partial cut harvesting and ectomycorrhizae: early effects in Douglas fir/Larch Forests of Western Montana. Can J For Res 10, 436-440

Harvey AE (1980b) Ecology of ectomycorrhizae in Northern Rocky Mountains forest. Envir Cons Timb Harc. In: RM Conif For Symp Proc, Missoula (1979) 189-208

Imbeck H, Ott E (1987) Verjüngungsökologische Untersuchungen im einem hochstaudenreichen subalpinen Fichtenwald, mit spezieller Berücksichtigung der Schneeablagerung und der Lawinenbildung. Mitt Eidg Inst Schnee und Lawinenforsch 82,202

Jacquiot C (1978) Écologie des champignons forestiers. Gauthier-Villars, Paris, $95 \mathrm{p}$

Kropp BR (1982a) Fungi from decayed wood as ectomycorrhizal symbionts of Western Hemlock. Can J For fies 12, 36-39

Kropp BR (1982b) Formation of Mycorrhizae on Nonmycorrhizal Western Hemlock outplanted on Rotten Wood and mineral soil. For Sci 28, 706-710

Lachaussée $E$ (1947) La régénération de l'épicéa en haute montagne. REF

Mac Cullough HA (1948) Plant succession on fallen logs in a virgin spruce-fir forest. Ecology 29, 508-513

Mac Millan PC, Means J, Hawk GM, Cromack J jr. Fogel R (1977) Log decomposition in an oldgrowth Douglas-fir forest. In: NW Sc Ass Abst Pap 50th Ann Meet. Pullman, USA, 13

Maser C, Anderson RG, Williams JT, Martin RE (1979) Dead and down woody material. In: Wildife Habitats in Managed Forests: The Blue Mountains of Oregon and Washington (Thomas Jack Ward, ed). Agric Handb 553, 78-95

Maser C, Trappe JM (1984a) The fallen tree a source of diversity. In: New Forests for a Changing World Proc Soc of Am For Natl Conf 335-339 
Maser C, Trappe JM (1984b) The Seen and Unseen World of the Fallen Tree. USDA, Forest Service, Report PNW 164, $56 \mathrm{p}$

Mathey A (1911) Régénération de l'épicéa dans les forêts des Hautes Régions. BSFFC 169

Mayer H (1976) Gebirgswaldbau-Schutzwaldlege. Ein Waldbaulichen Beitrag zur Landschaftökologie und zum Umweltschutz. Fischer, Stuttgart, $436 \mathrm{p}$

Moreau R, Poly J (1967) La régénération de l'épicéa dans les hautes chaînes du Jura. CR Acad Sci Paris 264, D, 1789-1 791
Moreau $R$, Poly $J$ (1968) La régénération de l'épicéa dans les hautes chaînes du Jura. BSFFC 1-2

Piussi P (1986) La rinnovazione della pecceta subalpina. Le Scienze 215, 58-67

Seidl MT (1985) The role of the fallen tree in an old-growth forest. In: 6th N Am Conf on Mycorrh. Bend, Oregon, 274

Sorg JP (1980) Végétation et rajeunissement naturel dans la pessière subalpine de Vals (GR). Mem Inst Fed Rech For 56, 116 